\title{
le sevrage tabagique par les dentistes et stomatologues
}

RÉSUMÉ La fumée du tabac est responsable de nombreuses maladies odontologiques, parondotologiques et stomatologiques car la bouche est en première ligne de l'exposition aux toxiques contenus dans la fumée.

Lors de soins, fumer expose au triplement du risque de complications du site opératoire, quel que soit le type de chirurgie: chirurgie générale, dents de sagesse ou implants. Arrêter de fumer 6-8 semaines avant une intervention chirurgicale supprime ce risque.

La prise en charge du tabagisme fait partie intégrante de l'exercice stomatologique. Tous les professionnels de la santé de la bouche doivent connaître le conseil minimal, la conduite à tenir en fonction du stade de préparation à l'arrêt et le maniement des substituts nicotiniques et des autres thérapeutiques d'aide à l'arrêt.

Bertrand DAUTZENBERG

Tabacologue,

Praticien hospitalier,

GH Pitié-Salpêtrière,

Assistance Publique - Hôpitaux de Paris,

Professeur à I'Université Médicale

Pierre et Marie Curie, Paris $\mathrm{VI}$

Président de l'Office Français

de prévention du Tabagisme (OFT)

www.oft-asso.fr

Coordinateur du réseau européen

des hôpitaux sans tabac (ENSH)

Service de Pneumologie

et Réanimation,

GH Pitié-Salpêtrière,

75651 Paris Cédex 13.
Par la fréquence des contacts qu'ils ont avec des sujets jeunes, les praticiens sont des acteurs importants de la lutte contre le tabac, qui se sont mobilisés dans de nombreux pays, mais peu encore en France, autour de ce thème de santé individuelle et de santé publique.

\section{MOTS CLÉS}

arrêt du tabac

substituts nicotiniques

conseil minimum

prévention 


\section{introduction}

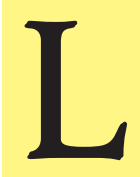

e tabagisme est une des causes prévenables de la détérioration de l'état buccodentaire.

Traiter le tabagisme est tout autant un acte odonto-stomatologique, que de traiter une douleur par antalgique ou traiter une infection par antibiotiques. Les praticiens ne dirigent vers un centre de la douleur qu'un infime pourcentage de leurs consultants douloureux, vers un généraliste ou un infectiologue qu'un infime pourcentage de leurs malades infectés ; il n'y a aucun raison qu'ils ne fassent pas de même pour le sevrage tabagique, qui bien souvent ne nécessite pas de prescription, mais seulement du conseil dans la mesure où les substituts nicotiniques sont disponibles sans prescription.

Le tabac a une toxicité faible par rapport à sa fumée. Si le tabac à chiquer ou à mâcher a une toxicité propre, la bouche est en première ligne de l'exposition à la fumée du tabac. La fumée du tabac est responsable de nombreuses maladies odontologiques, parodontologiques et stomatologiques.

Fumer expose au triplement du risque de complication du site opératoire[1], quel que soit le type de chirurgie, qu'il s'agisse de chirurgie étendue[1], d'extraction de dents de sagesse[2] ou de la pose d'implants $[3,4]$. La consolidation osseuse est particulièrement concernée et se fait trois fois plus souvent avec retard. Arrêter de fumer 6-8 semaines avant une intervention chirurgicale supprime ce risque. Tout acte chirurgical sur la bouche, même minime, doit dans la mesure du possible être une occasion d'arrêter de fumer, au minimum 6-8 semaines avant et jusqu'à la fin de la période de cicatrisation ( 3 semaines pour la peau et les muqueuses, 3 mois pour l'os) [1].

En fonction de sa compétence, chacun pourra aller plus ou moins loin dans la prise en charge, mais tous les spécialistes de la bouche doivent connaître le conseil minimal, la conduite à tenir en fonction du stade de préparation à l'arrêt et le maniement des substituts nicotiniques.

Par la fréquence des contacts qu'ils ont avec des sujets jeunes, les praticiens sont des acteurs importants de la lutte contre le tabac, regroupés dans de nombreux pays[5-12], mais pas encore en France autour de ce thème. En luttant contre le tabac, les professionnels améliorent la santé globale, diminuent aussi les maladies stomatologiques et réduisent les complications de leurs actes chirurgicaux.

\section{repérer le tabagisme et le stade de préparation à l'arrêt de chaque fumeur}

\section{- le conseil minimum}

Le minimum à faire pour tout professionnel est de poser la question «fumez- vous ?» Si la réponse est négative : féliciter ; si la réponse est positive demander au fumeur s'il envisage d'arrêter ? C'est ce que l'on appelle le conseil mini- 
mum[13]. Ce conseil minimum prend peu de temps et permet au patient de faire un lien entre son tabagisme et sa santé et peut contribuer, avec d'autres messages, à une décision d'arrêt, de maintien de l'arrêt ou de prévention des reprises et faire progresser le fumeur dans les stades de préparation au changement[13].

- le diagnostic de stade de préparation au changement

Di Clemente et Prochaska ont proposé un schéma de préparation au changement qui s'adapte parfaitement à la relation entre le fumeur et son tabagisme (fig. 1) et qu'il est aisé pour tout soignant de s'approprier.

Il est possible de classer les fumeurs dans un des stades de préparation afin de proposer une réponse adaptée à cha- cun de ces stades en posant la question "envisagez-vous d'arrêter de fumer ?" - Si la réponse est non, il faut prendre acte du refus, annoncer au patient le souhait de le voir arrêter de fumer un jour et dire que l'on est prêt à en reparler. Il est inutile d'insister plus à ce stade sauf si le tabagisme est associé à un problème médical ou qu'une intervention chirurgicale est programmée. Dans ces derniers cas on s'efforcera d'emporter l'adhésion du fumeur par un arrêt, au moins pour un temps, ou à défaut pour une réduction du tabagisme sous substituts nicotiniques. L'appel au tabacologue pourra être particulièrement utile.

- Si la réponse est oui, il faut essayer de préciser quand? Si le patient ne peut répondre que «un jour», il faut lui demander de dresser la liste des «avantages» et des «inconvénients à fumer». Le soignant doit reformuler la réponse $\mathrm{du}$ fumeur et si les inconvénients

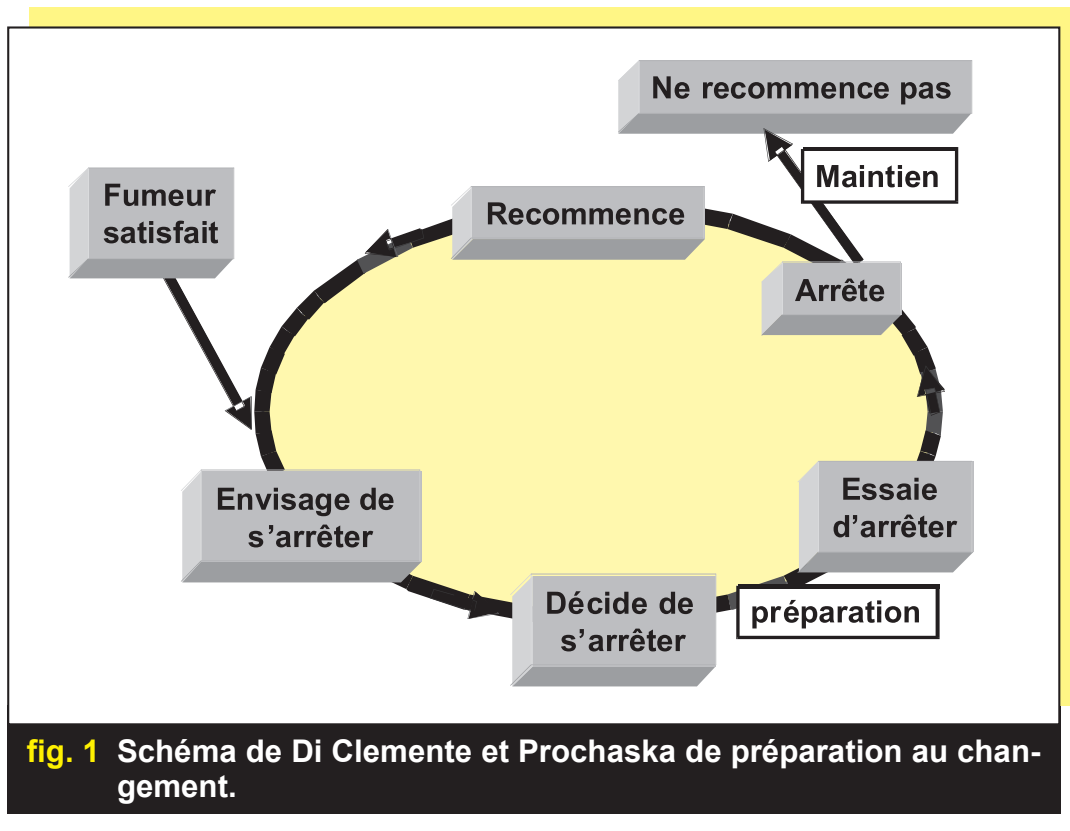


dépassent les avantages, lui proposer de fixer une période de l'année à venir pour s'arrêter ou une réduction du tabagisme sous substituts nicotiniques.

- Si le fumeur dit qu'il veut s'arrêter dans les 6 mois ou l'année à venir, il faut par le même entretien de motivation mettant en avant l'ambivalence du tabagisme, essayer de lui faire fixer une date précise d'arrêt dans 10 jours,... dans un mois.

- Si le fumeur est prêt à s'arrêter dans un délai court, il faut lui présenter les aides à l'arrêt disponibles, l'aide que l'on peut éventuellement lui apporter, les aides médicamenteuses utilisées en fonction de la dépendance à la nicotine. Si l'on peut arrêter seul, cela est plus facile, il y a moins d'effets secondaires et plus de chances de succès si l'on se fait aider.

- Si le fumeur a déjà arrêté depuis quelques jours ou semaines, il faudra prévenir les récidives. En effet, les reprises du tabagisme surviennent dans la moitié des cas dans l'année après un arrêt de 3 mois de tabagisme.

\section{- préciser le tabagisme}

Dans tous les cas, il est utile de reconstituer l'histoire du tabagisme, de préciser l'intensité de la consommation, les différents produits du tabac fumés (cigarettes industrielles, cigarettes roulées, cigarillos, cigares, pipes, chicha ou narguilé, bidis, (tabac + cannabis,...) et en ce qui concerne la pathologie buccodentaire les produits du tabac non fumés sont également sources de pathologies (snuff, snuss, tabac à chiquer,...).
Le dialogue doit aider à définir les motifs pour lesquels le fumeur consomme et expliquer l'importance d'un arrêt face aux éventuels problèmes de santé rencontrés actuellement ou qui pourraient survenir, de façon paradoxale des problèmes «mineurs» de santé, en particulier stomatologiques, peuvent être des soutiens de motivation plus forts qu'un risque d'accident de santé plus grave, qui reste moins concret pour le patient. Les tests de motivation et de réussite pourront être exploités chez ces patients.

\section{- dépendance tabagique}

Le mode de prise en charge sera adapté à la dépendance tabagique qui doit être précisée dans tous les cas. Toute dépendance est un phénomène complexe, où sont associés et imbriqués des mécanismes comportementaux et pharmacologiques : le tabagisme est un comportement entretenu et amplifié par une dépendance pharmacologique dont la nicotine est responsable.

- La dépendance comportementale est liée au geste, à l'habitude, à la pression sociale et à la convivialité.

- La dépendance psychique se manifeste par la recherche du plaisir, de la relaxation, de la détente, de la stimulation intellectuelle de la cigarette.

- La dépendance physique se traduit par un syndrome de manque lié à la privation de nicotine responsable de pulsions intenses à fumer (craving), d'irritabilité, d'anxiété, d'agitation et d'agressivité. Elle se mesure par le test de Fagerström (tableau I), questionnaire en 6 items dont le score total est de 10 points. La dépendance est 


\section{Tableau I}

Test de dépendance à la nicotine de Fagerström (traduction recommandations Afssaps).

\section{Combien de temps après votre réveil fumez-vous votre première cigarette ?}

3 Dans les 5 premières minutes

2 Entre 6 et 30 minutes

$1 \quad$ Entre 31 et 60 minutes

0 Après 60 minutes

2. Trouvez-vous difficile de vous abstenir de fumer dans les endroits où c'est interdit ?
1 Oui
0 Non

3. À quelle cigarette de la journée vous sera-t-il plus difficile de renoncer ?

1 La première le matin

$0 \quad$ N'importe quelle autre

4. Combien de cigarettes fumez-vous par jour?

010 ou moins

111 à 20

221 à 30

331 ou plus

5. Fumez-vous à un rythme plus soutenu le matin que l'après-midi ?

1 Oui

0 Non

6. Fumez-vous lorsque vous êtes malade, que vous devez rester au lit presque toute la journée?

1 Oui

0 Non

Score de $\mathbf{0}$ à 2 : le sujet n'est pas dépendant à la nicotine. Il peut arrêter de fumer sans avoir recours à des substituts nicotiniques. Si toutefois le sujet redoute cet arrêt, les professionnels de santé peuvent lui apporter des conseils utiles.

Score de 3 à 4 : le sujet est faiblement dépendant à la nicotine.

Score de 5 à $\mathbf{6}$ : le sujet est moyennement dépendant. L'utilisation des traitements pharmacologiques de substitution nicotiniques va augmenter ses chances de réussite. Le conseil du médecin ou du pharmacien sera utile pour l'aider à choisir la galénique le plus adapté à son cas.

Score de 7 à 10 : le sujet est fortement ou très fortement dépendant à la nicotine. L'utilisation de traitements pharmacologiques est recommandée (traitement nicotinique de substitution, varenicline ou bupropion L.P.). Ce traitement doit être utilisé à dose suffisante et adaptée. En cas de difficulté, orienter le patient vers une consultation spécialisée. 
nulle de 0 à 2 points, légère à 3 et 4 points, moyenne à 5 et 6 points et forte de 7 à 10 points.

Les items les plus importants portent sur le besoin de remonter le taux de nicotinémie le matin au réveil (à quel moment fumez-vous votre première cigarette ?) et le nombre de cigarettes fumées. Il est possible d'utiliser en pratique courante le test de Fagerström simplifié en 2 items (comportant la question 1 et 4 du test long) donnant ainsi un score sur 6 , facilement mémorisable.

\section{- les marqueurs du tabagisme}

L'analyse du CO dans l'air expiré se fait grâce à un appareil simple qui coûte environ 300 euros. Il permet une prise de conscience rapide au-delà du discours de la pollution par la fumée de tabac et de la disparition rapide de cette pollution à l'arrêt du tabagisme. L'analyse du CO de l'air expiré facilite la communication et valorise toute démarche d'arrêt temporaire ou de réduction du tabagisme sous substituts nicotiniques.

La quantification des marqueurs du tabagisme (HbCO lors de la mesure des gaz du sang, éventuellement cotinine ou autres dérivés de la nicotine) reflète sa consommation actuelle, mais n'est absolument pas indispensable à la prise en charge de la plupart des fumeurs.

- les recommandations pour la pratique clinique de l'Afssaps[13]

(Agence française de sécurité sanitaire des produits de santé)

Ces recommandations reprennent l'essentiel des recommandations pour l'arrêt et constituent un outil utile pour tous les acteurs du tabagisme.

Il est nécessaire pour tout consultant fumeur :

- de préciser la dépendance physique à partir des questions du test de Fagerström ;

- d'informer sur la dépendance comportementale : quels sont les gestes, les habitudes, les rituels, les circonstances qui font allumer une cigarette (café du matin, pause café...) ;

- de renforcer la motivation à l'occasion des soins, moment favorable ;

- de proposer les outils thérapeutiques les plus adéquats ;

- d'informer, d'expliquer et de définir une stratégie concertée d'arrêt du tabac en apportant des informations pertinentes et claires sur les effets nocifs $d u$ tabac et les avantages d'arrêter.

\section{traitement}

- les outils

de traitement du tabagisme

L'ensemble des outils d'aide à l'arrêt a été évalué dans la conférence de consensus sur l'arrêt du tabac de 1998[14], puis réévalué dans le document plus récent des recommandations pour la pratique clinique de l'Afssaps[13]. Outre le conseil minimum, les traitements comprennent : 


\section{LES THÉRAPIES COGNITIVES ET COMPORTEMENTALES (TCC)}

Approche récente en psychologie, les T.T.C. reposent sur l'apprentissage et l'analyse des pensées. Elles ont pour objectif d'aider le sujet à se débarrasser du comportement anormal par un nouvel apprentissage, tout en analysant les pensées ou «cognitions» qui accompagnent ce comportement.

\section{LA LIGNE D'AIDE TABAC INFO SERVICE (TIS)}

La ligne téléphonique d'aide à l'arrêt (Tabac Info Service 0825309310 , $0,15 €$ la minute) peut aider aussi bien les fumeurs que les soignants qui ont à prendre en charge un fumeur.

\section{LA SUBSTITUTION NICOTINIQUE}

La substitution nicotinique est indiquée chez le fumeur qui présente une dépendance physique à la nicotine, confirmée par le test de Fagerström.

Elle permet d'éviter le syndrome de manque des premières semaines de l'arrêt qui constitue chez de nombreux fumeurs un frein considérable à l'arrêt et une cause de rechute précoce fréquente.

De nombreuses études ont prouvé son efficacité puisqu'elle double presque le pourcentage de succès par rapport au placebo.

Pourtant, ces études ont été réalisées avec des schémas relativement rigides en terme de posologie et de durée de traitement, et par conséquence un probable sous-dosage chez les sujets fortement dépendants.

On peut raisonnablement penser qu'avec une adaptation très personnalisée des doses et de la durée du traitement, les résultats peuvent être considérablement améliorés.

Il existe actuellement 4 formes galéniques disponibles de substitution nicotinique.

\section{Les timbres ou "patchs»}

Sous 3 dosages différents, ils délivrent par voie transdermique une quantité contrôlée de nicotine à travers la peau. L'absorption de la nicotine se fait lentement et le plateau de nicotinémie recherché est obtenu deux heures après la pose $d u$ premier timbre.

Celui-ci doit être laissé en place pendant 16 ou 24 heures selon la marque et changé quotidiennement.

\section{Les gommes}

L'absorption de la nicotine délivrée par les gommes à mâcher à travers la muqueuse buccale permet de soulager le manque au bout de quelques minutes. La gomme doit être conservée dans la bouche pendant 20 à 30 minutes. Son utilisation nécessite le respect de quelques règles (la «croquer» une fois par minute, ne pas avaler trop rapidement sa salive,...) au risque de voir apparaître des effets désagréables (hoquets, épigastralgies). Son goût un peu poivré rebute parfois certains patients, on peut alors conseiller l'utilisation de gommes aromatisées à la menthe fraîche, à l'orange ou aux fruits.

\section{Les comprimés sublinguaux et à sucer}

Les comprimés sublinguaux et comprimés à sucer ont une cinétique d'absorption similaire à la gomme sans les inconvénients liés à la mastication et aux problèmes des gommes chez les patients aux prothèses non solidement fixées. 


\section{L'inhaleur}

L'inhaleur est une sorte de fume-cigarette contenant une cartouche poreuse imprégnée de nicotine.

L'absorption de la nicotine «aspirée» par le fumeur se fait par les muqueuses buccales, comme pour la gomme et le comprimé.

Son intérêt repose sur la reproduction de la composante gestuelle du comportement tabagique qui peut satisfaire certains fumeurs.

\section{CONDUITE DU TRAITEMENT DE SUBSTITUTION NICOTINIQUE}

La posologie initiale doit être adaptée aux besoins réels du fumeur, mesurés principalement par deux questions du test de Fagerström (tableau II) puis adaptée au bout de quelques jours.

La persistance de signes de manque nicotinique (pulsions à fumer, instabilité, anxiété,....) doit mener à augmenter la dose. Il est possible, chez les fumeurs très dépendants, d'administrer simultanément deux timbres (tableau II).

L'utilisation conjointe de plusieurs formes galéniques est possible (tableau II).

Par exemple, un timbre de $21 \mathrm{mg} / 24$ heures auquel on associe soit des gommes, soit des pastilles sublinguales pour répondre à un besoin plus important à certains moments de la journée (après les repas, lors d'un stress ou lors d'une situation conviviale).

Le surdosage est, lui, rare et transitoire. Il se manifeste par l'absence totale d'envie de fumer, la sensation d'avoir trop fumé, la bouche pâteuse, parfois nausées et palpitations.

Il est recommandé que le fumeur choisisse lui-même la date de début de son traitement, certains préférant un weekend calme de repos à une période de stress professionnel, d'autres favorisent le choix inverse.

Tableau II

Posologie initiale des substituts nicotiniques en fonction du score de Fagerström simplifié.

\begin{tabular}{|c|c|c|c|c|}
\hline Fume & $\leq 10 \mathrm{cig} . / \mathrm{j}$ & $11-20 \mathrm{cig} . / \mathrm{j}$ & 21-30 cig./j & $>30$ cig./j \\
\hline $\begin{array}{c}+60^{\prime} \text { après } \\
\text { le lever }\end{array}$ & $\begin{array}{l}\text { Rien ou formes } \\
\text { orales }\end{array}$ & $\begin{array}{l}\text { Formes orales } \\
\text { et/ou timbre } \\
\text { (moyen) }\end{array}$ & $\begin{array}{l}\text { Timbre (grand) } \\
\text { et/ou formes } \\
\text { orales }\end{array}$ & $\begin{array}{c}\text { Timbre (grand) } \\
\pm \text { formes } \\
\text { orales }\end{array}$ \\
\hline $\begin{array}{c}30 \text { à } 60^{\prime} \text { après } \\
\text { le lever }\end{array}$ & Formes orales & $\begin{array}{l}\text { Timbre (moyen } \\
\text { ou grand) et/ou } \\
\text { formes orales }\end{array}$ & $\begin{array}{l}\text { Timbre (grand) } \\
\pm \text { formes orales }\end{array}$ & $\begin{array}{c}\text { Timbre (grand) } \\
+ \text { formes } \\
\text { orales }\end{array}$ \\
\hline $\begin{array}{c}<30^{\prime} \text { après } \\
\text { le lever }\end{array}$ & $\begin{array}{c}\text { Timbre (moyen) } \\
\text { ou } \\
\text { formes orales }\end{array}$ & $\begin{array}{l}\text { Timbre (moyen } \\
\text { ou grand) } \pm \\
\text { formes orales }\end{array}$ & $\begin{array}{l}\text { Timbre (grand) }+ \\
\text { formes orales }\end{array}$ & $\begin{array}{c}\text { Timbre(s) } \\
\text { (grand +/- } \\
\text { moyen) }+ \\
\text { formes orales }\end{array}$ \\
\hline $\begin{array}{c}<5^{\prime} \text { après } \\
\text { le lever }\end{array}$ & $\begin{array}{l}\text { Timbre (moyen) } \\
\pm \text { formes orales }\end{array}$ & $\begin{array}{l}\text { Timbre (grand) } \\
+ \text { formes orales }\end{array}$ & $\begin{array}{c}\text { Timbre(s) } \\
\text { (grand +/- } \\
\text { moyen) } \\
+ \text { formes orales }\end{array}$ & $\begin{array}{c}\text { Timbres } \\
\text { (grand }+ \\
\text { moyen) }+ \\
\text { formes orales }\end{array}$ \\
\hline
\end{tabular}


Le suivi est ensuite programmé à un rythme adapté à chaque fumeur. Certains demandent un soutien très rapproché, d'autres pas. Le suivi permet d'adapter les doses de substitution, la décroissance étant la plus progressive possible.

La réapparition de signes de manque, au moment d'un passage à une dose inférieure, impose d'augmenter celle-ci pour quelques jours ou semaines avant d'envisager une nouvelle diminution. La durée du traitement sera fonction de la dose de substitution administrée au départ. Initialement prévu pour trois mois, le traitement pourra atteindre cinq ou six mois chez les sujets les plus dépendants.

\section{Le bupropion}

Le Zyban ${ }^{\circledR}$ (bupropion LP) est un inhibiteur de la recapture de la dopamine et de la noradrénaline. Son efficacité sur l'arrêt du tabac a d'abord été notée de manière empirique dans les années 1990. Il double les chances d'arrêter de fumer. Les effets secondaires les plus fréquemment relevés dans les essais cliniques ont été : bouche sèche, insomnie, céphalée. Le risque de convulsion avait été évalué à $1 / 1000$ chez les sujets traités par la molécule pour dépression. Cependant, les situations cliniques augmentant le risque de convulsion doivent être considérées comme des contre-indications à la prescription de ce médicament.

Le traitement débute par un comprimé de $150 \mathrm{mg}$ pendant 6 jours, puis un comprimé matin et soir de $150 \mathrm{mg}$, en respectant un intervalle de huit heures entre les prises. La durée du traitement est de 7 à 9 semaines. L'arrêt total du tabac doit être décidé entre le 7 e et le $14 \mathrm{e}$ jour du traitement.
L'accompagnement et le soutien $d u$ fumeur pendant toute la durée du traitement, et au-delà pour prévenir les rechutes sont, comme pour les substituts nicotiniques, des éléments indissociables de la prise en charge.

\section{La varenicline (Champix ${ }^{\circledR}$ )}

C'est un inhibiteur de sous-récepteurs ß4a2 nicotiniques qui a, sur les essais disponibles, un effet supérieur aux autres médicaments existants.

\section{Autres traitements}

L'acupuncture, l'homéopathie, la mésothérapie, l'hypnose et les autres méthodes à effet essentiellement placebo ont été, depuis longtemps, utilisées pour le sevrage tabagique. Leur efficacité chez un fumeur motivé non fortement dépendant tient à l'effet placebo qu'il délivre et à l'empathie délivrée par le thérapeute. La Conférence de Consensus d'octobre 1998[14], et les recommandations de l'AFSSAPS en mai 2003[13] ne leur ont pas reconnu d'efficacité sur la dépendance nicotinique.

\section{- mise en œuvre du sevrage en stomatologie \\ LORS D'UN EXAMEN BUCCO-DENTAIRE DE PRÉVENTION}

Questionner sur le tabagisme fait partie intégrante de l'examen bucco-dentaire de prévention. Énoncer l'intérêt chez les enfants non fumeurs de rester non fumeurs est un renforcement pour donner l'image saine d'une vie sans tabac. 


\section{LORS D'UN SOIN}

\section{STOMATOLOGIQUE ORDINAIRE}

Donner le conseil minimum est la règle chez tous les fumeurs, alors que des enquêtes récentes montre que seul $28 \%$ des praticiens américains le font[5]. Il est recommandé que les spécialistes fassent plus et organisent une aide aux fumeurs, qu'ils peuvent réaliser eux-mêmes, ou les diriger vers d'autres professionnels de santé ou la ligne d'aide à l'arrêt Tabac Info Service.

\section{LORS D'UNE INTERVENTION CHIRURGICALE}

Pour les spécialistes pratiquant des actes chirurgicaux de la bouche, extraction de dents de sagesse, implants ou interven- tions plus larges, l'organisation de l'arrêt du tabac est un impératif. Cet arrêt est au mieux obtenu 6-8 semaines avant l'intervention chirurgicale. Il justifie que chaque spécialiste s'organise pour dépister les fumeurs plus de 8 semaines avant l'intervention et organise une prise en charge des fumeurs pour l'arrêt ou à défaut pour la réduction du tabagisme sous substitution nicotinique partielle. Les consultations de tabacologie, la ligne d'aide à l'arrêt Tabac Info Service (0 825309 310) et les autres professionnels de santé pourront aider les praticiens à définir la meilleure stratégie de prise en charge pour ces fumeurs afin de minimiser les complications des interventions bucco-dentaires ou stomatologiques pratiquées.

\section{conclusions}

Les praticiens sont des acteurs naturels de la lutte contre le tabac. Ils peuvent et doivent jouer pleinement leur rôle de professionnels de santé. Comme les autres professionnels de santé ils peuvent prendre eux-mêmes une part plus ou moins large du sevrage tabagique, mais ils doivent dans tous les cas conduire le conseil minimum et orienter les fumeurs qu'ils ne veulent pas prendre en charge eux-mêmes. Que ce soit à l'échelon individuel ou à l'échelon de la santé publique, cette prise en charge est bénéfique.

\section{bibliographie}

1. Dureuil B, Dautzenberg B, Masquelet AC. Tabagisme péri-opératoire. Presse Med 2006;35: 1009-1015.

2. Larsen PE. Alveolar osteitis

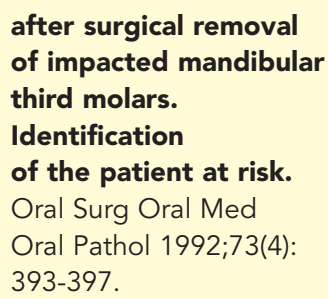

3. Ellegaard B, Baelum V, Kolsen-Petersen J. Non-grafted sinus implants in periodontally compromised patients: a time-to-event analysis. Clin Oral Implants Res 2006;17:156-164 (RR=2.2) 
4. Vehemente VA, Chuang SK, Daher S, Muftu A,

Dodson TB.

Risk factors affecting

dental implant survival.

J Oral Implantol 2002;28:

74-81.

5. Albert DA, Severson $H$, Gordon J, Ward A,

Andrews J, Sadowsky D.

Tobacco attitudes,

practices, and behaviors:

a survey of dentists

participating

in managed care.

Nicotine Tob Res 2005;

7(Suppl 1):S9-18.

6. Christen AG, Christen JA,

Charles G. Pease, DDS, MD

Anti-tobacco crusader

and clean life advocate.

J Hist Dent 2001;49:81-86.

7. Albert DA, Severson $H$, Gordon J, Ward A,

Andrews J, Sadowsky D.

Tobacco attitudes,

practices, and behaviors:

a survey of dentists

participating in managed care.

Nicotine Tob Res 2005;

7(Suppl 1):S9-18.

8. Newton JT

Encouraging patients

to quit.

Br Dent J 2006;201:99.

9. Stacey $F$, Heasman PA, Heasman L, Hepburn S, McCracken GI, Preshaw PM.

Smoking cessation as a dental intervention Views of the profession. Br Dent J 2006;201: 109-113.

10. Dyer TA, Robinson PG. General health promotion in general dental practice The involvement of the dental team.

Part 1: a review

of the evidence

of effectiveness of brief public health interventions.

Br Dent J 2006;200

679-685.

11. Watt RG.

Health promotion by the dental team.

Br Dent J 2006;200:671.

12. Albert DA, Severson HH, Andrews JA.

Tobacco use by adolescents: the role of the oral health professional in evidencebased cessation programs. Pediatr Dent 2006;28: 177-187.

13. Recommandations de bonne pratique. AFSSAPS.

Les stratégies

thérapeutiques médicamenteuses et non médicamenteuses de l'aide à l'arrêt du tabagisme. Alcool Addictol 2003; 25(2 suppl):1S-44S.

14. Conférence de consensus : arrêt de la consommation du tabac,

1998.

http://www.sante.gouv.fr/ htm/pointsur/tabac/5tab21. htm\#4

\section{Role of dentists and stomatologists in smoking suspension}

\section{B. DAUTZENBERG}

Tobacco smoke is a cause for many teeth, parondotologic and stomatologic diseases because the mouth is in first line of the exposure to the toxics contained in smoke.

At the time of care, to smoke expose to three-fold increase of the risk of complications of the surgical site, as in other surgical: procedure, wisdom teeth extraction, implants. Stop smoking 6-8 weeks before surgical operation eliminates this risk. To take care of smoking is a part of the stomatologic treatment. All health professional of the mouths has to know the minimal counselling, the action to be taken according to the course of preparation to change and the handling of the nicotine replacement therapy and the other therapeutic of smoking control.

By the frequency of the contacts with young subjects, the clinicians are significant actors of the fight against the tobacco, which were mobilized in many countries, but not in France, around this individual and public health concern.

keywords: smoking cessation, nicotin replacement therapy, minimum counselling, prevention. 